\title{
First results of the Focusing DIRC prototype, an innovative detector for charged particle identification
}

\author{
M. Borsato ${ }^{* a}$, N. Arnaud ${ }^{b}$, B. Dey ${ }^{c}$, K. Nishimura ${ }^{d}$, D.W.G.S. Leith ${ }^{e}$, D. Roberts ${ }^{f}$, \\ B. Ratcliff ${ }^{e}$, J. Va'vra $^{e}$, G.S. Varner ${ }^{d}$ \\ ${ }^{a}$ Univ Paris Diderot, Sorbonne Paris Cite, Paris, France \\ ${ }^{b}$ LAL, UMR 8607 Univ Paris-Sud, CNRS/IN2P3, Orsay, France \\ ${ }^{c}$ University of California Riverside, CA 92521, USA \\ ${ }^{d}$ University of Hawaii, Honolulu, 96822, USA \\ eSLAC National Accelerator Laboratory, Stanford, CA 94309, USA \\ ${ }^{f}$ University of Maryland, College Park, MD 20742, USA \\ E-mail: borsatodlal.in2p3.fr
}

The FDIRC (Focusing Detector of Internally Reflected Cherenkov light) is a charged particle identification (PID) detector which separates efficiently kaons from pions up to a few $\mathrm{GeV} / \mathrm{c}$.

It is a ring-imaging Cherenkov detector based on the same concept as the BABAR DIRC. Yet, its design has been significantly improved to be able to operate at much higher luminosity $\left(10^{36} \mathrm{~cm}^{-2} \mathrm{~s}^{-1}\right)$ and with larger backgrounds. Indeed, the FDIRC was intended to cover the barrel region of the SuperB detector, a new generation flavour factory cancelled at the end of 2012 due to lack of funding.

The new design consists in new compact photon cameras made out of fused silica which are instrumented with highly-pixelated Hamamatsu H-8500 MaPMTs and readout by new fast frontend digitizing electronics. A full-scale prototype of a FDIRC sector (1/12th of the whole detector) has been successfully built at SLAC and is taking data in the Cosmic Ray Telescope facility since the beginning of 2013 .

In this contribution, we briefly review the FDIRC design and the construction of the SLAC prototype. Then, we focus on the challenges associated with the FDIRC data analysis which is aided by a detailed Geant4-based simulation of the test facility. Finally, we summarize the status of the ongoing data taking phase and present the first preliminary results based on data.

The European Physical Society Conference on High Energy Physics -EPS-HEP2013

18-24 July 2013

Stockholm, Sweden

\footnotetext{
${ }^{*}$ Speaker.
} 


\section{Introduction}

Detectors for experiments studying the flavour sector of the standard model require very good Particle IDentification (PID) performances. This is why, as opposed to general-purpose particle physics detector, their design often includes a device dedicated to PID. Most of these devices are based on the visible Cherenkov light emitted by a charged particle when traversing a refractive medium at a speed larger than the speed of light in the medium.

A very successful Cherenkov PID detector has been the DIRC (Detector of Internally Reflected Cherenkov light) [1], a barrel PID device that has been crucial to the performance of the BABAR physics programme. However, this detector could not perform well in a very high luminosity machine such as the ones that are being designed nowadays to look for new physics in very rare decays. Indeed, a higher luminosity brings a higher background, which requires a redesign of the device. Therefore, based on the concept of the BABAR DIRC, a R\&D project was started to develop the Focusing DIRC (FDIRC), a faster and much more compact PID device which can deal with a background 100 times higher [2,3]. The FDIRC was intended to cover the barrel region of the SuperB experiment, a project which was cancelled at the end of 2012 due to lack of funding.

\section{The FDIRC concept}

The weak point of the BABAR DIRC was its huge imaging camera filled of water which was very sensible to electromagnetic and neutron backgrounds. In order to reduce dramatically the sensitivity to background the camera has been completely redesigned to be much smaller and faster. The invaluable BABAR DIRC quartz bars are instead reused, requiring the new camera to be consistent with them. The new design is modular: it consists of 12 solid blocks machined from radiation-hard pieces of fused silica in place of the large water-filled camera.

The requirement of reducing the volume of the cameras to the minimum led to a new imaging concept in which the Cherenkov ring is focused in the vertical direction by a cylindrical mirror (Fig. 1). Also, the ring is folded several times by total reflection on the sides of the camera to fit on a smaller plane instrumented with Photo-Multiplier Tubes (PMT). These small cameras require fine photon detector pixelation: several multi-anode PMTs were considered for the final design of the FDIRC, but finally the Hamamatsu H-8500 MaPMT was selected [4]. By design, each camera is instrumented with $48 \mathrm{H}-8500$ MaPMTs having 64 pixels each. The pixels are horizontally grouped two by two to reduce the total number of channels; this has no impact on the Cherenkov angle resolution which is determined mainly by the vertical coordinate of the hits.

The H-8500 MaPMT meets also the requirement of a time resolution as low as $\sim 0.2 \mathrm{~ns}$ (the $B A B A R$ DIRC had a resolution of $1.7 \mathrm{~ns}$ ). This is required to reduce the sensitivity to background by about one order of magnitude. Furthermore, this very good timing performance is expected to provide an improvement of the Cherenkov angle resolution by using a measurement of the chromatic dispersion term in the radiator and the separation of ambiguous solutions in the folded optical system.

Further details about the design can be found in the SuperB Technical Design Report [5]. 

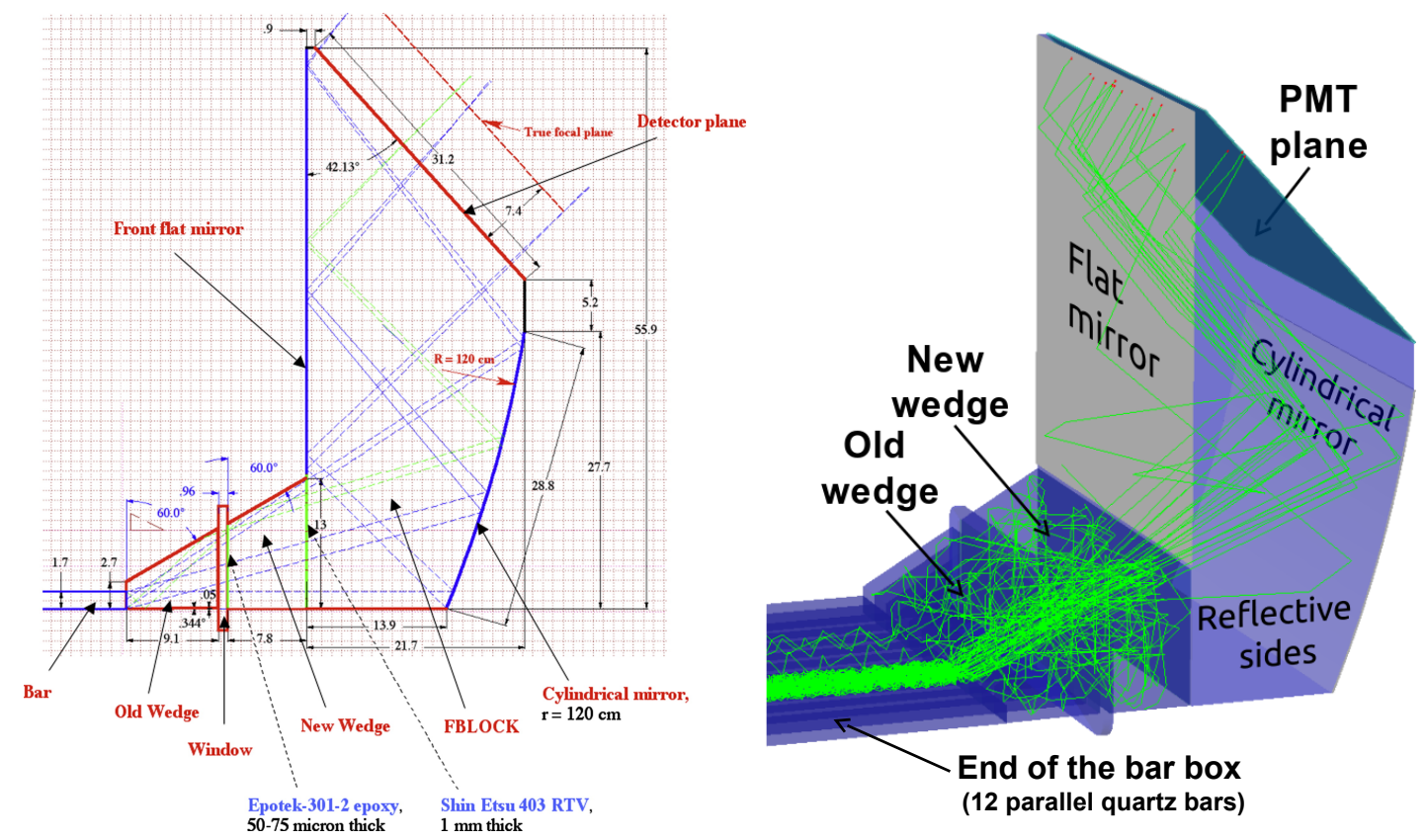

Figure 1: (left) FDIRC optical design (dimensions are in $\mathrm{cm}$ ). Dashed lines show photon paths from the bar exit to the detector plane where they are focused onto. All paths include reflections on the two mirrors. The green photon path includes also a reflection on the top of the wedge. (right) A 3-D sketch of the photon camera from the Geant 4 simulation. The paths of Cherenkov photons from a charged muon track are represented in green. Few of them got reflected on the interface between the different quartz pieces. These photons may result on a PMT hit, but they are significantly delayed and can easily be rejected.

\section{First full-size FDIRC sector at the SLAC CRT}

After the successful running of a first prototype consisting of an oil-filled camera [6,7], the final optics for one sector was built [8] to validate the FDIRC design for the SuperB experiment. A new wedge and FBLOCK were machined out of fused-silica with a surface-polishing quality of $30 \AA$ RMS. Then, the FBLOCK, the new wedge and the box containing the 12 DIRC bars were coupled with optical glue.

The first full-size sector of the FDIRC is installed at the SLAC Cosmic Ray Telescope (CRT) as shown in Fig. 2. This facility [9] is well suited for testing this kind of detectors since it can select hard cosmic muons $\left(E_{\mu}>2 \mathrm{GeV}\right)$ with a tracking position resolution of $\sim 5 \mathrm{~mm}$, a tracking angular precision of $\sim 1.5 \mathrm{mrad}$, and very good timing thanks to a Cherenkov start-counter. Furthermore, its large angular acceptance provides 3-D tracks and not just one-directional tracks as an electronbeam.

The FDIRC prototype is equipped with $12 \mathrm{H}-8500$ MaPMTs (out of the 48 of the original design). They are placed in the PMT plane region where most Cherenkov photons are expected. The signal from each pixel passes through a SLAC amplifier card which provides a factor 40 of voltage gain. Then, it is digitized by an IRS2 ASIC [10], a fast waveform sampler configured to sample at $\sim 2.7 \mathrm{GSa} / \mathrm{s}$. The digitized data are sent to a back-end CompactPCI ${ }^{\circledR}$ (cPCI) crate via gigabit fiber links; a linux-based DAQ system running on a cPCI CPU collects the digitized waveforms and stores them to disk for off-line analysis. 


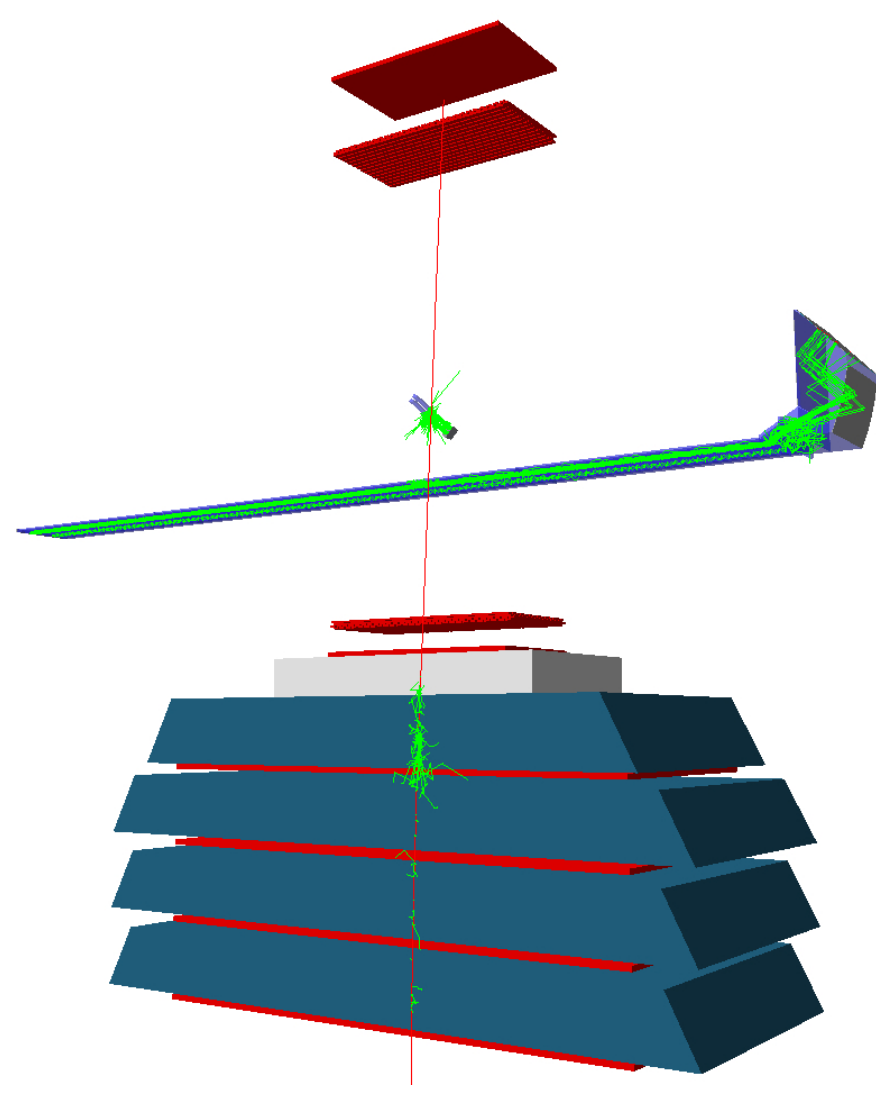

Figure 2: Schematic view of the FDIRC prototype installed in the CRT from the Geant 4 simulation. High energetic cosmic muons (red track in the figure) are used as a probe to test the detector. The simulation is as realistic as possible: it includes all the sub-detectors composing the CRT.

\section{Data analysis and simulation}

A very accurate Geant4-based simulation of the FDIRC detector was developed [11]. It has been a fundamental tool to estimate the performances of the full barrel detector at the time it was designed. Lately, a new version of the code was developed to simulate the whole experimental setup with the full-scale sector of the FDIRC installed at the CRT. All the relevant parts composing the CRT are simulated also and cosmic muons are generated with the proper distribution in terms of angles and energies (Fig. 2). This allows us for extensive studies of real data and the understanding of systematics.

Furthermore, such an accurate simulation is fundamental to the data-analysis software since it is used to produce look-up tables that map PMT pixel positions to photon directions $(\hat{\mathbf{k}})$ at the generation point inside the bars [12]. These tables are constructed firing millions of single photons in each bar with random directions and collecting the corresponding PMT hits. The definition of the different photon paths is done clustering events coming from close enough directions $\hat{\mathbf{k}}_{i}$. Note that the complex folded optics of the FDIRC results in many possible photon directions for each PMT pixel position. In particular, looking at Fig. 1, one realizes that there are many possible paths from a bar exit to a pixel. Indeed the photon can go "directly" to the PMT plane or bounce on one 


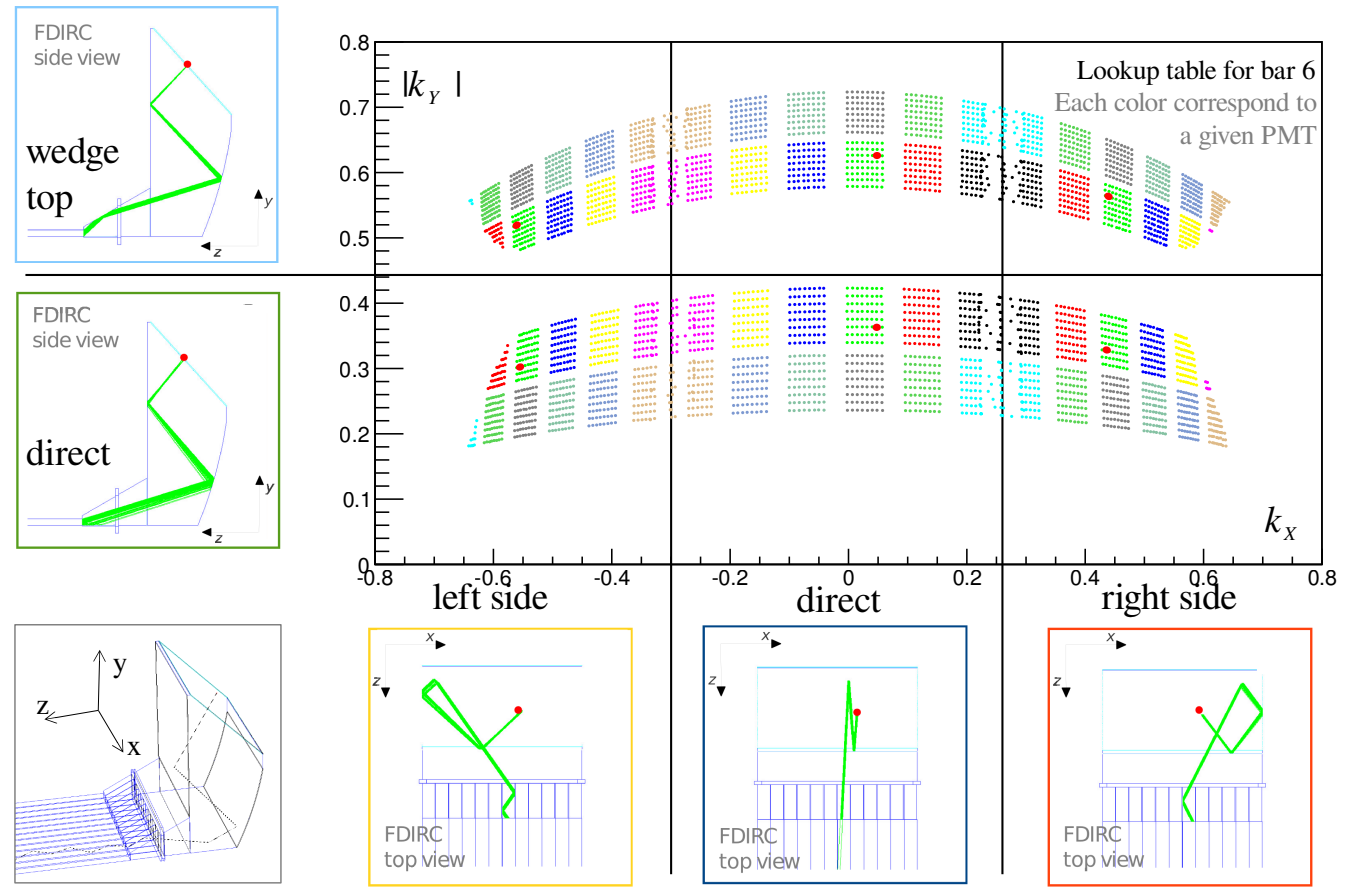

Figure 3: In the centre, a plot of all the possible directions $\hat{\mathbf{k}}$ from the exit of bar number 6 (in the middle) to each of the $12 \times 64$ pixels. The directions of pixels belonging to different PMTs are represented with dots of different colours. Note that the image of the instrumented plane (composed of 12 PMTs) is mirrored on the left and on the right. Then, this 3 -fold image is reflected on the top resulting in a total of $3 \times 2=6$ images of the PMT plane. The classification of these 6 images is explained by the two pictures on the left and the three on the bottom which show sketches of the different types of photon paths inside the FBLOCK. In the bottom left is just an explication of the reference frame used.

of the FBLOCK sides. On top of that, each of these paths is doubled because the photon can either bounce on the top surface of the wedge or not. This makes a total of 6 paths per bar per pixel. A way of visualizing and understanding the complex folding is shown in Fig. 3.

The classification of the photon paths and the generation of the look-up table were subjects of extensive studies. In particular there is a complication due to the fact that the old wedge is horizontally split in 12, while the new wedge is not (Fig. 1 right). For this reason, the best way of building the table is to cluster the signed value of $k_{X}$ taken at old wedge exit: indeed, in the $x$ direction the old wedge has reflective surfaces along with the ones of the bars. At the same time, we cluster the absolute value of $k_{Y}$ at bar exit since both old and new wedge have a reflective surface at the top inclined by $60^{\circ}$.

This is not the complete picture though: since the number of bounces the photon did in the bar is unknown, there is also a sign ambiguity on all the three components $\pm k_{X}, \pm k_{Y}$ and $\pm k_{Z}$ of the direction of the photon at generation $\hat{\mathbf{k}}$. Therefore, the FDIRC design produces as many as $6 \times 8=48$ ambiguities for each pixel hit. Still, the FDIRC is able to choose the right solution for most hits thanks to its very good timing capabilities and taking advantage of the information on the charged track direction given by the CRT. Indeed, the analysis software calculates the expected Time Of Propagation (TOP) for each possible path, compares it to the real hit time and discards 
solutions whit a large difference of the two. Some ambiguities, though, have just the opposite sign of $k_{X}$ or $k_{Y}$ and therefore the same expected TOP; yet, they normally give non-physical values for $\theta_{C}$, so they can be identified and rejected.

Furthermore, there is another way good timing improves the Cherenkov angle resolution of the FDIRC: the application of the so-called chromatic correction. Indeed, the chromatic contribution to the Cherenkov angle resolution is reducible through a measurement of the group velocity of the photon in the quartz. Thanks to the very long path length of photons in the bar (some metres) and to the very precise timing $(\sim 0.2 \mathrm{~ns})$ the group velocity and thus the group index of refraction can be measured very precisely. Then, knowing the dispersion relation in the quartz, one can infer the phase index $n_{\mathrm{ph}}$, whose dependence on the wavelength is responsible for the chromatic broadening of the Cherenkov angle. Studies with the first oil-filled prototype showed that chromatic correction can improve the Cherenkov angle resolution by $0.5-1.0 \mathrm{mrad}$ if a time resolution as low as $\sim 0.2 \mathrm{~ns}$ is reached $[13,14]$.

\section{Conclusion}

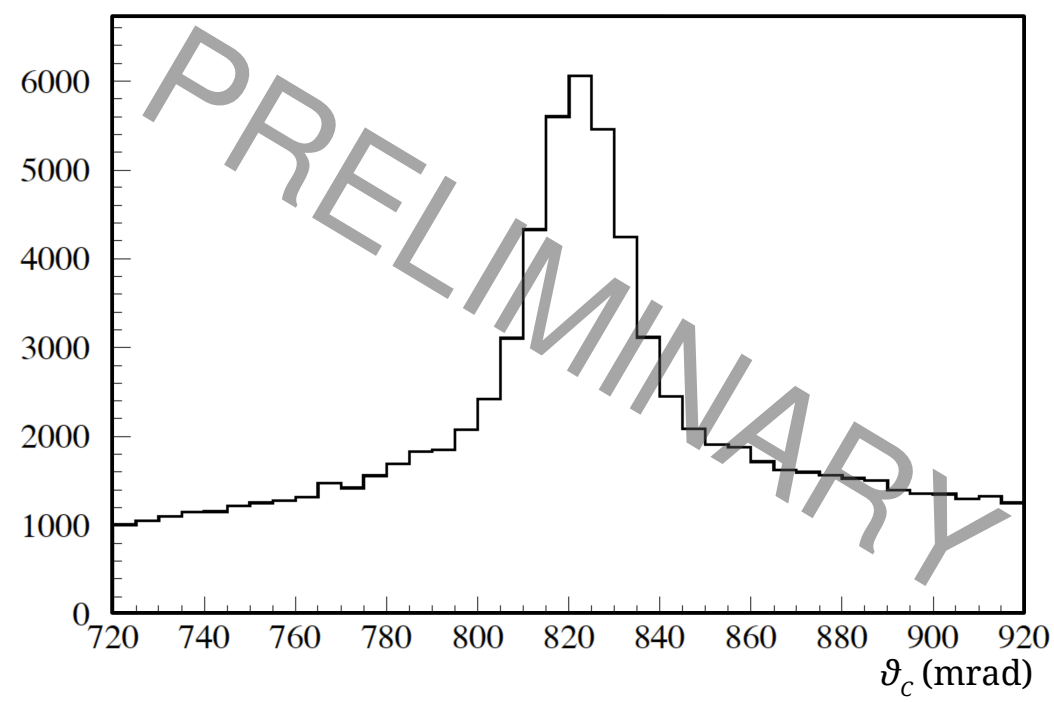

Figure 4: Preliminary result for the distribution of the Cherenkov angle measured by the FDIRC prototype installed at the SLAC CRT. No chromatic correction nor electronics calibration was applied to produce this plot. In particular the time alignment is expected to greatly improve the signal to noise ratio. Nevertheless, the clear peak at the expected value of $\theta_{C} \sim 822 \mathrm{mrad}$ validates the optical design.

This R\&D project was successful in building the first full-size FDIRC prototype despite all technical challenges. The detector is now being tested at the SLAC Cosmic Ray Telescope facility. Data taking is ongoing and final results are expected by the end of the year. Preliminary results are very encouraging as can be seen in the plot of the Cherenkov angle peak for $2 \mathrm{GeV}$ muons in Fig. 4. This clear peak at the expected Cherenkov angle ( $\sim 822 \mathrm{mrad}$ for muons with $E>2.0 \mathrm{GeV}$ ) validates the optical design with 3-D tracks. Note that the background, rather than being completely random, is mainly due to unresolved ambiguities on the photon direction. No quantitative performance results are shown here, but early numbers are very encouraging and al- 
ready close to expectations from the Geant 4 simulation. Yet, we expect to greatly improve this result by a better timing alignment which is being carried out using a pulse injected into the dynode of each PMT. This should result in a dramatic increase of the signal to noise ratio and in a better $\theta_{C}$ resolution thanks to the chromatic correction. Also, the data analysis software is being developed and may change in the future. Final results will be available later this year, namely the single photon Cherenkov angle resolution, the number of detected photons per ring, the pollution given by unresolved ambiguities and finally the effect of the chromatic correction.

\section{Acknowledgments}

We thank SLAC technician M. McCulloch for his excellent work done. We would also like to thank M. Benettoni for his excellent work concerning the mechanical support structure for the FDIRC optics. The author of these proceedings would like to thank the France-Stanford Center for Interdisciplinary Studies for its financial support.

\section{References}

[1] I. Adam et al., The DIRC particle identification system for the BaBar experiment, Nucl. Instr. Meth. A538 (2005) 281-357.

[2] J. Va'vra, SLAC-PUB-13464, 2008, and Focusing DIRC design for SuperB , SLAC-PUB-13763, 2009.

[3] J. Va'vra, D. Roberts and B. Ratcliff, FDIRC design for SuperB , Nucl.Instr. Meth. A404 (2011) 282.

[4] C. Field et al., Development of Photon Detectors for a Fast Focusing DIRC, Nucl. Instr. and Meth. A553 (2005) 96-106.

[5] SuperB Collaboration, SuperB Technical Design Report, arXiv:1306.5655v1.

[6] L.L. Ruckman et al., The focusing DIRC with waveform digitizing electronics, Nucl. Instr. and Meth. A623 (2010) 303-305.

[7] K. Nishimura et al., A detailed study of FDIRC prototype with waveform digitizing electronics in cosmic ray telescope using 3D tracks, Nucl. Instr. and Meth. A701 (2013) 115-126.

[8] J. Va'vra et al., Progress on development of the new FDIRC PID detector, Nucl. Instr. and Meth. A718 (2013), 541-545.

[9] J. Va’vra, SLAC Cosmic Ray Telescope Facility, 2009, report SLAC-PUB-13873.

[10] L.L.Ruckman, G.S. Varner, and A. Wong, Nucl. Instr. and Meth. A591 (2008) 534.

[11] D. Roberts, FDIRC reconstruction, in 3rd SuperB Collaboration Meeting. March, 2012.

[12] M. Borsato et al., The Focusing DIRC: an innovative PID detector, Nucl. Instr. and Meth. A (2013) DOI:10.1016/j.nima.2013.08.014 .

[13] J. Benitez et al., Status of the Fast Focusing DIRC (FDIRC), Nucl. Instr. and Meth. A595 (2008) 104, and SLAC-PUB-12236.

[14] J. Va'vra et al., The Focusing DIRC - the First RICH Detector to Correct the Chromatic Error by Timing, and the Development of a New TOF Detector Concept, SLAC-PUB-1280, 2007. 